\title{
Efeitos de Registros de Automonitorização sobre Relatos de Adesão ao Tratamento em Adolescentes com Lúpus
}

\author{
Flávia Pinho Almeida ${ }^{1}$ \\ Eleonora Arnaud Pereira Ferreira \\ Ana Júlia Pantoja de Moraes \\ Universidade Federal do Pará
}

\begin{abstract}
RESUMO - Descreve-se o estudo realizado com duas adolescentes (P1 e P2) com diagnóstico de lúpus acompanhadas em ambulatório de reumatologia. Investigaram-se os efeitos do uso de registros de automonitorização (RA) e de recordatório 24 horas (R24h) sobre o índice de adesão ao tratamento (IAT) por meio de entrevistas semiestruturadas. P1 foi submetida à sequência R24h-RA e P2, à ordem inversa. Em linha de base, ambas obtiveram valores de IAT abaixo ou igual a 50\%. Após intervenção, alcançaram valores acima de $71 \%$, indicando melhora no índice de adesão independentemente da ordem em que os instrumentos foram apresentados. Discute-se o controle do comportamento por regras e a importância da instalação de repertórios de relatos fidedignos sobre comportamentos de adesão ao tratamento do lúpus
\end{abstract}

Palavras-chave: psicologia da saúde, lúpus, adesão ao tratamento, automonitorização.

\section{Effects of Self-Monitoring Records on Reports of Treatment Adherence in Adolescents with Juvenile Lupus}

\begin{abstract}
This article describes a study conducted with two teenagers (P1 and P2) who were diagnosed with lupus and attended a rheumatology clinic. The aim was to investigate the effects of the use of a self-monitoring registry (RA) and a $24 \mathrm{~h}$ recall (R24h) on the rate of Treatment Adherence Index (IAT) through semi-structured interviews. Participant P1 was submitted to a 24h-RA sequence whereas participant P2 to the reverse order. At baseline, both of them had IAT rates below or equal to $50 \%$; after the intervention, they achieved rates above $71 \%$, showing improvements in adherence. The control of behavior by rules and the importance of installing a set of reliable accounts of behavior of adherence to the treatment of lupus were discussed.
\end{abstract}

Keywords: health psychology, lupus, treatment compliance, self-monitoring.

O American College of Reumatology (ACR, 2008) considera o lúpus eritematoso sistêmico como uma doença autoimune, caracterizada por inflamação crônica e difusa por todo o corpo. Quando diagnosticado em crianças e adolescentes é denominado de lúpus eritematoso sistêmico juvenil (LESJ). Na apresentação juvenil, essa enfermidade chama atenção por comprometer gravemente os rins, demandando imediata intervenção (Sociedade Brasileira de Reumatologia, 2011).

A terapêutica para o LESJ inclui um conjunto de instruções as quais dependem de cada organismo e da severidade da manifestação (Pongmarutani, Alpert, \& Miller, 2006; Sociedade Brasileira de Reumatologia, 2011). Entretanto, é comum a prescrição de medicamentos (como corticoides, antimaláricos, imunossupressores, analgésicos e anti-inflamatórios); a recomendação para evitar exposições prolongadas ao sol, com o uso de hidratante e de filtro solar várias vezes ao dia; além de alimentação saudável, que contenha pouco sal e gordura. $\mathrm{O}$ tratamento requer, portanto,

1 Endereço para correspondência: Universidade Federal do Pará, Núcleo de Teoria e Pesquisa do Comportamento, Cidade Universitária Prof José da Silveira Netto, Campus do Guamá, Rua Augusto Corrêa 1, Belém, PA, Brasil. CEP: 66.175-110. E-mail: eleonora_arnaud@yahoo.com.br mudanças significativas no estilo de vida dos pacientes, o que tem implicado em baixos índices de adesão (Duvdevany, Cohen, Minsker-Valtzer, \& Lorber, 2011).

Aderir ao tratamento é um fator imprescindível para a melhora do estado clínico do paciente com LESJ. Na área de saúde, a adesão é analisada de acordo com a correspondência entre as instruções de cuidado prescritas pelos profissionais de saúde e os comportamentos emitidos pelo paciente, a partir de acordos estabelecidos durante as consultas (World Health Organization [WHO], 2003). Sob a perspectiva da análise do comportamento, comportamentos de adesão ao tratamento - tais como tomar medicamentos, seguir orientações quanto a mudanças na alimentação e prática de exercícios físicos - podem ser mantidos por reforçamento negativo, uma vez que consistem na fuga ou esquiva dos sintomas da doença, assim como por reforçamento positivo, pois a emissão de tais respostas pode ter como consequência imediata a aprovação social. Desse modo, tais comportamentos fazem parte de uma classe operante, uma vez que se trata de um conjunto de respostas que opera sobre o ambiente e que produz consequências funcionalmente equivalentes, sendo modelados e mantidos pela interação de regras e suas consequências (Malerbi, 2000).

Regras são estímulos antecedentes verbais que podem descrever contingências (Skinner, 1974) e exercer múltiplas 
funções (Albuquerque, 2001). Comportar-se de acordo com regras significa que o indivíduo se comporta de uma forma que foi determinada por outro indivíduo que especificou as contingências (Albuquerque \& Paracampo, 2010; Sousa, Paracampo, \& Albuquerque, 2015). De acordo com Matos (2001), regras são particularmente importantes quando se trata de situações em que as contingências naturais são fracas ou operam em longo prazo.

Uma vez que ainda são poucos os estudos experimentais sobre controle do comportamento por regras na área da saúde, tem-se utilizado pesquisas experimentais realizadas em laboratórios de análise do comportamento como referência para compreender a relação entre instruções de profissionais de saúde e adesão ao tratamento pelo paciente. Por exemplo, estudos como os de Barret, Deitz, Gaydos e Quinn (1987), Cerutti (1994) e Hayes et al. (1985) sugerem que o comportamento de seguir instruções tende a ser mantido pela presença de membros de uma comunidade verbal, chamados de autoridade, que monitoram o seguimento de tais regras. No caso de crianças e adolescentes com doenças crônicas, essas autoridades seriam os cuidadores e os profissionais de saúde, uma vez que estes auxiliam na monitorização do tratamento. Entretanto, alguns obstáculos relacionados às características do próprio tratamento, aos comportamentos do paciente e aos fatores sociais podem fazer-se presentes (Malerbi, 2011).

No LESJ, os comportamentos de adesão devem ser emitidos regularmente durante um extenso período de tempo, ou mesmo por toda a vida; no entanto, os resultados desse tratamento podem demorar a aparecer. Nesses casos, Ferreira e Fernandes (2009) destacam que, uma vez que as consequências não reforçam de imediato os comportamentos de adesão, é necessário que um mediador social auxilie o paciente a identificar as relações de controle então vigentes. Como o tratamento do LESJ produz perda de reforçadores (e.g., a reeducação alimentar, a restrição à atividade física), e mesmo a exposição a estímulos aversivos (efeitos colaterais da medicação, como edemas faciais e queda de cabelo), a presença do cuidador ou da equipe de saúde monitorando o comportamento do paciente pode auxiliar a instalar e a manter o comportamento de seguir as instruções do tratamento, auxiliando inclusive a criança e o adolescente a gerir o próprio tratamento, como demonstrado no caso do diabetes Tipo 1 (Mulvaney et al., 2010; Palmer et al., 2010).

$\mathrm{Na}$ área da saúde, estudos que utilizaram o referencial teórico da análise aplicada do comportamento obtiveram resultados positivos apontando a eficácia do uso de registros de automonitorização (RA) sobre a instalação de comportamentos de seguir regras para o tratamento. Tais estudos apontam que houve melhora na adesão ao tratamento após a introdução do uso de RA, tanto em pacientes adultos (e. g., Casseb \& Ferreira, 2012; Casseb, Bispo-Malcher, \& Ferreira, 2008; Ferreira \& Fernandes, 2009) quanto em crianças (e.g., Hidaka, 2007; Martins, Ferreira, Cavalcante, \& Almeida, 2015). A automonitorização corresponde ao comportamento de observar e registrar, sistematicamente, a ocorrência de determinado comportamento pelo próprio indivíduo (Bohm \& Gimenes, 2008), o que pode favorecer a aquisição de repertórios de auto-observação, melhorando o autoconhecimento do paciente, que é decisivo para a mudança comportamental favorável aos objetivos terapêuticos (Barton, Blanchard, \& Veazey, 1999).

Outro instrumento também utilizado é o recordatório 24 horas (R24h). Neste, o indivíduo é solicitado a descrever os comportamentos de adesão emitidos nas últimas 24 horas, permitindo-lhe discriminar as situações em que realizou ou deixou de realizar alguma orientação prescrita pelo profissional (Bueno \& Czepielewski, 2010; Ribeiro, Costa, Sobral, \& Florindo, 2011). Assim, tanto o RA quanto o R24h podem ser considerados como instrumentos de controle do comportamento a partir da apresentação de regras. Entretanto, ainda são poucos os estudos nessa área, em especial voltados para a população com LESJ, o que justifica a realização deste estudo.

Neste estudo, investigaram-se os efeitos do uso de RA e do R24h sobre relatos de adesão ao tratamento de duas adolescentes com diagnóstico de LESJ atendidas entre agosto de 2012 e julho de 2013 no ambulatório de reumatologia pediátrica do Hospital Universitário Bettina Ferro de Souza (HUBFS), da Universidade Federal do Pará. Buscou-se caracterizar o comportamento de adesão ao tratamento antes da intervenção e verificar se o uso de RA e R24h, com apresentação de feedback, aumentaram a ocorrência de relatos de adesão ao tratamento.

\section{Método}

O presente estudo experimental teve por base o delineamento intra-sujeito, longitudinal de curto prazo, com duas condições experimentais (Condição 1 - R24h-RA e Condição 2 - RA-R24h) para eliminar o efeito de ordem na apresentação dos instrumentos. Dessa forma, as participantes foram inseridas em uma dentre as duas condições de acordo com a ordem de entrada no estudo. As análises dos dados obtidos no decorrer dos meses de intervenção e acompanhamento de cada participante foram realizadas tendo por base o sujeito como seu próprio controle.

\section{Participantes}

Participaram do estudo duas adolescentes (P1 e P2) com LESJ encaminhadas pela médica reumatologista pediátrica do ambulatório do HUBFS para fazerem parte da pesquisa por apresentarem dificuldades de adesão ao tratamento. Ambas residiam na região metropolitana de Belém-PA e aceitaram participar da pesquisa, assinando, juntamente com os seus respectivos responsáveis, o termo de assentimento e o de consentimento livre e esclarecido (Projeto aprovado pelo CEP/ICS/UFPA, CAEE 0262.0.073.073-12, Protocolo No. 001/12). Foram excluídos do estudo os pacientes recémdiagnosticados, os que residiam em outro município e aqueles que compareceram à consulta com demanda de atendimento de urgência. 


\section{Instrumentos}

Formulário de observação da consulta médica. Contendo colunas e linhas onde eram registradas as instruções (regras) apresentadas pela médica reumatologista durante a consulta, possibilitando a descrição fidedigna do tratamento prescrito para cada participante.

Roteiro de Entrevista de Pós-Consulta. Elaborado para este estudo, por meio do qual foram obtidas informações a respeito da consulta ocorrida, do tratamento proposto pela médica e das possíveis facilidades e dificuldades que cada participante julgou que iria enfrentar para realizar o tratamento indicado.

Recordatório 24 horas. Roteiro de entrevista por meio do qual foi solicitado que as participantes descrevessem os comportamentos de adesão ao tratamento emitidos durante o intervalo de 24 horas antecedentes à entrevista, assim como o contexto em que ocorriam esses comportamentos.

Formulário de Registro de Automonitorização. Instrumento elaborado a partir das instruções prescritas pela médica a cada participante durante a consulta observada. Composto por uma coluna contendo a lista de comportamentos indicados ao tratamento que deveriam ser seguidos pela participante no intervalo entre consultas e mais setes colunas para o registro das ocorrências desses comportamentos no período de uma semana.

Roteiro de Entrevista com Feedback. Continha a lista de instruções prescritas pela médica durante a consulta ambulatorial observada, assim como as informações relatadas pela participante no Roteiro de Entrevista de Pós-Consulta, e a representação gráfica dos relatos de adesão a cada instrução obtidos até o momento da entrevista, sendo utilizado para fazer a análise da consistência entre as regras prescritas para o tratamento e o relato de seguimento dessas regras.

Roteiro de Entrevista Final. Elaborado com o objetivo de apresentar a cada participante os resultados alcançados com a intervenção.

\section{Procedimento}

As duas adolescentes indicadas pela médica reumatologista à pesquisa foram abordadas enquanto aguardavam em sala de espera, juntamente com seus responsáveis, pela consulta de rotina no ambulatório do HUBFS. O convite foi feito mediante a leitura e assinatura dos termos de consentimento e de assentimento.

Inicialmente, fez-se o acompanhamento e registro de uma consulta médica com cada participante, utilizando-se o Formulário de Observação da Consulta Médica e um gravador de áudio pela pesquisadora, a qual se manteve a uma distância adequada para não intervir durante o atendimento, mas em local que lhe permitia observar a interação da médica e da participante. Em seguida, fez-se a aplicação do Roteiro de Entrevista de Pós-Consulta e do R24h com cada participante, por meio do qual foi feito o levantamento de seu repertório de entrada ou Linha de Base 1. Após o intervalo de uma semana, foi realizada outra entrevista com cada participante utilizando-se o R24h para obtenção da Linha de Base 2.
A partir de então, P1 e P2 foram divididas, respectivamente, entre a Condição 1 (R24h-RA) e a Condição 2 (RA-R24h), iniciando a fase de intervenção do estudo. $\mathrm{Na}$ Condição 1 , foi apresentado primeiro o R24h e, em seguida, o Formulário de RA junto com o R24h. Na Condição 2, foi invertida a ordem de apresentação dos instrumentos, iniciando-se com o Formulário de RA junto com o R24h, finalizando com a apresentação apenas do R24h.

A fase de intervenção foi realizada por meio de 10 entrevistas semanais, gravadas em áudio, com duração média de 60 minutos. Conforme acordo estabelecido, com P1, as entrevistas aconteceram no ambulatório do hospital e, com $\mathrm{P} 2$, em seu domicílio. Na Condição 1, as primeiras cinco entrevistas com P1 foram realizadas utilizando-se somente o R24h. Na quinta entrevista, foi-lhe apresentado o Roteiro de Entrevista com Feedback e, em seguida, a participante recebeu instruções para o preenchimento do Formulário de RA. Desse modo, do sexto ao décimo encontro, houve o acompanhamento do preenchimento do Formulário de RA juntamente com a aplicação do R24h a cada entrevista. Na Condição 2, as cinco primeiras entrevistas com P2 foram realizadas a partir do preenchimento do Formulário de RA acompanhado do R24h, com a apresentação do Roteiro de entrevista com feedback ao final do quinto encontro. Do sexto ao décimo encontro, fez a utilização somente do R24h.

Durante a realização de todas as entrevistas de intervenção, a terapeuta-pesquisadora apresentava elogios e gestos de aprovação como consequência aos comportamentos de registros e relatos de $\mathrm{P} 1$ e de $\mathrm{P} 2$ que fossem considerados como consistentes com as instruções prescritas para seus tratamentos. No caso de registros e relatos que não coincidiam com tais instruções, a terapeuta-pesquisadora elogiava as participantes por relatarem tais comportamentos durante a entrevista, destacando a importância de relatos fidedignos para o melhor planejamento do tratamento.

Paralelamente à realização das entrevistas de intervenção, fez-se a coleta dos dados contidos no prontuário de cada participante. Por fim, fez-se uma entrevista com a médica reumatologista para avaliação do quadro clínico de cada participante. Foi também realizado um encontro final com cada participante com a utilização de mais um Roteiro de Entrevista com Feedback e a aplicação do Roteiro de Entrevista Final.

\section{Análise de Dados}

Os dados obtidos a partir da aplicação dos R24h (10 para cada participante) foram quantificados, atribuindo-se o valor 1 para os casos em que a participante relatasse seguir o tratamento de acordo com as instruções dispostas pela médica e o valor 0 aos casos de não seguimento correto dessas instruções. A somatória da pontuação obtida foi transformada em percentual do Índice de Adesão ao Tratamento (IAT), pela seguinte equação:

\section{IAT $=\underline{n^{0} \text { de instruções seguidas } \times 100}$ $\mathrm{n}^{\mathrm{o}}$ total de instruções médicas}

Os dados referentes aos Formulários de RA (quatro para cada participante) também foram quantificados em 1 ou 0 , seguindo os mesmos critérios utilizados na análise dos R24h. 
Posteriormente, foram transformados em valores numéricos para uma melhor visualização dos resultados.

A entrevista com a médica reumatologista realizada no final da coleta de dados permitiu a obtenção da descrição do quadro clínico das participantes e do valor do Systemic Lupus Erythematosus Disease Activity Index (SLEDAI). Trata-se de um sistema quantitativo de avaliação da atividade do lúpus que possibilita ao médico averiguar se o tratamento que vem sendo prescrito ao paciente está sendo eficaz, com escore variando entre 0 (nenhuma característica de manifestação da doença) e 105 (manifestação de todas as características da doença em seus estados mais graves) (Katz, 2011).

\section{Resultados}

Observa-se na Tabela 1 que, ao início do estudo, o IAT das participantes ficou abaixo (P1) ou igual (P2) a 50\%. Esses resultados indicam que as participantes relataram o não seguimento de algumas instruções de tratamento prescritas pela médica, independentemente do tempo de diagnóstico ou do número de consultas já realizadas. Ambas também apresentavam bom controle do LESJ mediante os resultados dos exames médicos.

De acordo com os relatos de P1 e de P2, ambas tinham mais dificuldade no seguimento de instruções relativas à reeducação alimentar e ao uso de protetor solar e de hidratante. Ambas relataram melhor seguimento das instruções referentes ao tratamento medicamentoso, mesmo que de modo irregular.

Na Tabela 2, observa-se que, em Linha de Base 2, o IAT de P1 diminuiu de valor. A partir da terceira entrevista de intervenção, houve aumento do IAT, mantendo-se acima de $50 \%$ nas entrevistas subsequentes com a utilização exclusiva do R24h. O valor de IAT tornou-se mais elevado a partir da terceira semana de preenchimento do Formulário de RA combinado com o R24h (oitava entrevista de intervenção), chegando a assumir valores de 85,7\%. Mesmo assim, observou-se que essa participante não relatou em nenhuma das entrevistas $100 \%$ de adesão ao tratamento.

No caso de P2, observou-se aumento no IAT logo na segunda entrevista de linha de base. Tal resultado manteve-se

Tabela 1. Características das participantes P1 e P2 ao início do estudo

\begin{tabular}{|c|c|c|}
\hline Variáveis & P1 & $\mathbf{P 2}$ \\
\hline Idade & 12 anos & 14 anos \\
\hline Escolaridade & $7^{\circ}$ ano $/ 9$ & $9^{\circ}$ ano/9 \\
\hline Tempo de diagnóstico & 18 meses & 33 meses \\
\hline Número de consultas já realizadas no HU & 6 consultas & 18 consultas \\
\hline Exames clínicos: marcações fisiológicas & Normais & Normais \\
\hline Exames de laboratório: FAN ${ }^{\mathrm{a}}$, anti-Sm${ }^{\mathrm{b}}$, anti-DNA ${ }^{\mathrm{b}}$, anti-Ro ${ }^{\mathrm{c}}$, anti- $\mathrm{La}^{\mathrm{c}}$ & Negativos & Negativos \\
\hline SLEDAI $^{\mathrm{d}}$ & 0 & 0 \\
\hline Cuidador principal & Pai & Mãe \\
\hline IAT obtido em Linha de base 1 & $43 \%$ & $50 \%$ \\
\hline
\end{tabular}

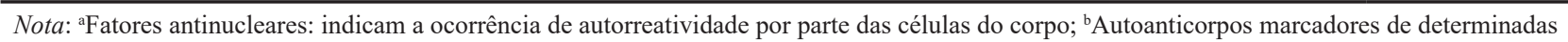
doenças; 'Autoanticorpos marcadores de atividade e/ou manifestação clínica da doença; ${ }^{\mathrm{d} S y s t e m i c ~ L u p u s ~ E r y t h e m a t o s u s ~ D i s e a s e ~ A c t i v i t y ~ I n d e x ~}$

Tabela 2. Valores do Índice de Adesão ao Tratamento de P1 e P2 em Linha de Base e após Intervenção

\begin{tabular}{llllll}
\hline P1 & \multicolumn{5}{l}{ P2 } \\
\hline Entrevistas & & IAT & Entrevistas & & IAT \\
\hline Linha de Base & 1 & $42,9 \%$ & Linha de Base & 1 & $50 \%$ \\
& 2 & $28,6 \%$ & & 2 & $75 \%$ \\
Recordatório 24h & 1 & $14,3 \%$ & Automonitorização + & 1 & $62,5 \%$ \\
& 2 & $42,9 \%$ & Recordatório & 2 & $62,5 \%$ \\
& 3 & $57,1 \%$ & & 3 & $62,5 \%$ \\
Automonitorização + & 6 & $71,4 \%$ & Recordatório 24h & 4 & $62,5 \%$ \\
Recordatório 24h & 7 & $71,4 \%$ & & 5 & $75 \%$ \\
& 5 & $71,4 \%$ & & 7 & $87,5 \%$ \\
& 8 & $85,7 \%$ & & 8 & $87,5 \%$ \\
& 9 & $71,4 \%$ & & 9 & $87,5 \%$ \\
& 10 & $85,7 \%$ & & 10 & $87,5 \%$ \\
\hline
\end{tabular}


Tabela 3. Instruções para tratamento prescritas pela médica e percentual de relatos de seguimento dessas instruções por P1 e P2 ( $n=10$ sessões) a partir da análise dos Recordatórios 24 horas

\begin{tabular}{llc}
\hline Instruções prescritas à P1 & & $\%$ \\
\hline Uso de Medicamentos & Forma de administração & \\
Prednisona & 01 comprimido $-60 \mathrm{mg} / 50 \mathrm{mg} / 40 \mathrm{mg} / 35 \mathrm{mg} / 20 \mathrm{mg} / 05 \mathrm{mg}$ (redução da & $80 \%$ \\
posologia durante o estudo)após café da manhã & 01 comprimido-25mg- de $12 \mathrm{~h}$ em $12 \mathrm{~h}$ & $90 \%$ \\
Captopril & 01 comprimido-50mg-à noite & $100 \%$ \\
Losartan & Forma de seguimento & $80 \%$ \\
Exercícios físicos/terapêuticos & Diariamente & $60 \%$ \\
Caminhada & Forma de seguimento & $40 \%$ \\
Proteção de pele & Aplicar no corpo todo após o banho ou todas as vezes que recordar & \\
Protetor solar & Aplicar no corpo todo todas as noites & $\%$ \\
Hidratante & & \\
& & $100 \%$ \\
Instruções prescritas à P2 & Forma de administração & $90 \%$ \\
\hline Uso de Medicamentos & 01 comprimido -05mg- de segunda à quinta após café da manhã \\
Ácido fólico & 01 comprimido -500mg- antes do almoço & $70 \%$ \\
Cálcio & 01 comprimido -200mg- depois do almoço & $90 \%$ \\
Hidroxicloroquina & 01 comprimido à noite & $50 \%$ \\
Losartan & 02 gotas antes de dormir & $100 \%$ \\
Adetil & Forma de seguimento & $20 \%$ \\
Proteção de pele & Aplicar no corpo todo após os banhos eno rosto no intervalo da escola & \\
\hline Hidratante & Aplicar no corpo todo todas as noites & \\
\hline
\end{tabular}

com a utilização conjunta do Formulário de RA e do R24h. Mesmo com a suspensão do Formulário de RA, o valor do IAT aumentou, chegando a assumir o valor máximo e, posteriormente, estabilizando-se em $87,5 \%$ nos três últimos encontros. Comparando-se a ordem de apresentação dos instrumentos, observa-se que os IATs obtidos por P2 foram mais elevados do que os obtidos por P1 ao longo da intervenção.

A Tabela 3 apresenta os resultados referentes ao percentual de relatos de seguimento para cada uma das instruções médicas prescritas para P1 e P2 ao longo das 10 sessões de intervenção em que somente o R24h foi utilizado.

Observa-se que, para ambas as participantes, as instruções para o tratamento medicamentoso foram aquelas que obtiveram melhores percentuais de seguimento. A exceção ficou restrita ao uso de vitamina A e D pela participante P2, cujo resultado indicou baixa adesão. A instrução de menor adesão foi a referente ao uso de hidratante, observada também em ambas as participantes.

Na Tabela 4, estão os resultados obtidos por P1 e P2 a partir da análise dos registros de adesão ao tratamento feita nos quatro Formulários de RA utilizados durante sete dias de quatro semanas. Foram consideradas as instruções referentes ao uso de medicamentos, realização de exercícios físicos/ terapêuticos e proteção de pele. Excluiu-se a análise dos registros referentes à reeducação alimentar uma vez que, durante o estudo, nenhuma das duas participantes havia se consultado com a nutricionista do ambulatório.
Observa-se que, no caso de $\mathrm{P} 1$, o número de registros de comportamentos de adesão ao tratamento foi menor nas duas primeiras semanas. A partir da segunda semana de registro, todas as orientações tiveram maior frequência de registro, culminando com melhor adesão na quarta semana. Novamente, os comportamentos com registros de adesão mais baixos foram o uso de protetor solar e do hidratante. No caso do protetor solar, P1 registrou que não fez uso nos turnos vespertino e noturno durante a primeira semana. Entre os medicamentos, o uso de Prednisona foi o mais regular, seguido de Losartan, ambos indicados para serem ingeridos uma única vez ao dia. Tanto o Carbonato de Cálcio quanto o Captopril, indicados para duas vezes ao dia, foram os medicamentos que apresentaram adesão irregular ao longo das quatro semanas de registro.

No caso de P2, observa-se também que o comportamento com menor registro de adesão foi o uso do hidratante. Quanto ao protetor solar, P2 registrou menor adesão ao uso deste em ambiente escolar. P2 declarou que fez uso do protetor solar em domicílio, principalmente no turno da manhã. Em relação ao uso de medicamentos, o Carbonato de Cálcio foi aquele cuja instrução foi seguida corretamente. Quanto aos demais medicamentos, houve boa adesão ao Metotrexato e ao Losartan após a terceira semana; enquanto o uso de Hidroxicloroquina e de vitaminas A e D permaneceu irregular durante as quatro semanas.

No decorrer de todo o estudo, durante a aplicação dos R24h e do Roteiro de Entrevista com Feedback, algumas 
Tabela 4. Registros de adesão ao tratamento observados nos Formulários de automonitorização dos participantes P1 e P2

\begin{tabular}{|c|c|c|c|c|c|c|}
\hline \multirow{2}{*}{ Turno } & \multirow{2}{*}{ Tratamentos } & \multicolumn{4}{|c|}{ Número de registros de adesão ao tratamento } & \multirow{2}{*}{$\Sigma$} \\
\hline & & Semana 1 & Semana 2 & Semana 3 & Semana 4 & \\
\hline \multicolumn{7}{|c|}{ Participante 1} \\
\hline \multirow[t]{4}{*}{ Manhã } & Predinizina & 7 & 7 & 7 & 7 & 28 \\
\hline & Captopril & 7 & 7 & 7 & 7 & 28 \\
\hline & Carbonato de cálcio & 5 & 7 & 7 & 7 & 26 \\
\hline & Protetor solar & 5 & 6 & 7 & 7 & 25 \\
\hline \multirow[t]{3}{*}{ Tarde } & Carbonato de cálcio & 6 & 6 & 7 & 6 & 25 \\
\hline & Exercício físico & 5 & 6 & 7 & 6 & 24 \\
\hline & Protetor solar & 0 & 5 & 6 & 7 & 18 \\
\hline \multirow[t]{4}{*}{ Noite } & Captopril & 6 & 6 & 6 & 7 & 25 \\
\hline & Losartan & 6 & 7 & 7 & 7 & 27 \\
\hline & Protetor solar & 0 & 4 & 6 & 7 & 17 \\
\hline & Hidratante & 2 & 5 & 6 & 7 & 20 \\
\hline \multicolumn{7}{|c|}{ Participante 2} \\
\hline \multirow[t]{4}{*}{ Manhã } & Ácido fólico ${ }^{\mathrm{a}}$ & 3 & $--^{c}$ & 7 & 4 & 14 \\
\hline & Metotrexato $^{\mathrm{b}}$ & 0 & - & 1 & 1 & 2 \\
\hline & Protetor solar-casa & 6 & - & 7 & 7 & 20 \\
\hline & Protetor solar-escola & 1 & - & 1 & 4 & 6 \\
\hline \multirow[t]{4}{*}{ Tarde } & Hidroxicloroquina & 6 & - & 7 & 5 & 18 \\
\hline & Metotrexatob & 0 & - & 1 & 1 & 2 \\
\hline & Cálcio & 7 & - & 7 & 7 & 21 \\
\hline & Protetor solar & 6 & - & 7 & 0 & 13 \\
\hline \multirow[t]{3}{*}{ Noite } & Losartan & 6 & - & 7 & 7 & 20 \\
\hline & Adetil & 6 & - & 6 & 6 & 18 \\
\hline & Hidratante & 0 & - & 1 & 4 & 5 \\
\hline
\end{tabular}

Nota: ${ }^{a}$ Medicamento com a instrução de ser utilizado por quatro dias na semana (de segunda a quinta-feira). ${ }^{\text {b} M e d i c a m e n t o ~ i n t r o d u z i d o ~ n a ~ t e r c e i r a ~ s e m a n a ~}$ com a instrução para ser ingerido em um único dia por semana. ${ }^{\mathrm{P}} \mathrm{P} 2$ não realizou o preenchimento do Formulário de automonitorização durante a segunda semana.

dificuldades foram relatadas pelas participantes, com destaque para a aplicação do protetor solar e a ingestão de alimentos gordurosos para P1. Para P2, obtiveram destaque as dificuldades relacionadas à restrição alimentar, a aplicação do protetor solar e a vida social. Quanto ao uso do protetor solar, essas dificuldades foram justificadas pelo "esquecimento" (P1 relatava que não tinha o hábito de levar o produto para a escola diariamente), assim como por "não gostar de sentir a pele oleosa após a aplicação do produto", de acordo com relatos de ambas as participantes (consequência aversiva imediata decorrente do uso do protetor solar). Quanto às restrições alimentares, P1 descreveu a dificuldade de recusar a ingestão de bacon, frequentemente preparado e oferecido por sua mãe (alimento gorduroso, não recomendado pela médica e disponível à jovem em sua residência). P2 relatou a dificuldade de não poder comer o que "todas as pessoas normais comem em festas", o que também comprometia a qualidade de sua vida social, pois $\mathrm{P} 2$ descreveu que recusava convites de passeios com os primos e com as amigas.

No entanto, também houve relatos de instruções médicas facilmente seguidas pelas participantes, como foi o caso da caminhada para $\mathrm{P} 1$, atividade que já era considerada como habitual. A administração de medicamentos foi descrita por ambas como fácil de seguir devido já "fazer parte de suas rotinas".

Ao serem solicitadas a avaliar a aplicação dos R24h e dos Formulários de RA, ambas afirmaram que se sentiram beneficiadas com essas intervenções, como se pode observar nas seguintes falas: "Foi bom, porque foi aí que eu comecei a me lembrar mais dos remédios... essas coisas" (P1 - em relação aos R24h); "Eu achei que foi bom também porque me ajudou", "Pra eu poder me lembrar dos remédios também... essas coisas", "foi fácil fazer em casa" (P1 - em relação aos exercícios de automonitorização); "legal", "ajudou a ver que eu não passava o hidratante" (P2 - em relação aos R24h); e "eu gostei", "ajudou a lembrar sim... quando eu esquecia, eu lembrava quando pegava o papel", "é que toda hora eu lembrava quando via aquele papel" (P2 - em relação aos exercícios de automonitorização).

\section{Discussão}

Neste estudo, verificou-se que o uso de RA e do R24h esteve associado à melhora nos IATs de duas adolescentes com LESJ quando comparados com os valores obtidos 
em linha de base. Tais instrumentos podem ter favorecido a instalação de repertórios de auto-observação e de autoconhecimento (Bohm \& Gimenes, 2008) referentes à adesão ao tratamento, independentemente da ordem em que os instrumentos foram apresentados.

Os dados obtidos demonstraram a complexidade do tratamento do LESJ, uma vez que as instruções médicas incluíam a associação de diversas classes de respostas. A complexidade desse conjunto de instruções confirma estudos que apontam para a dificuldade de seguimento de regras quando estas são extensas e as contingências naturais operam em longo prazo (Albuquerque \& Paracampo, 2010; Matos, 2001).

Um dos possíveis motivos para ambas as participantes apresentarem dificuldades na aplicação regular do protetor solar e do hidratante era o fato de esses comportamentos estarem sob o controle de um esquema de reforçamento negativo atrasado. Isto é, a consequência natural esperada seria o não aparecimento de manchas vermelhas e de estrias na pele (comuns em indivíduos com LESJ), efeito observado somente após longo período de uso dos produtos. Esse fato, somado ao relato de não gostarem de sentir a pele oleosa logo em seguida à aplicação desses produtos (consequência aversiva imediata), criava uma contingência propícia para o não seguimento dessas regras. Tais resultados corroboram estudos experimentais que apontam que o seguimento de regras é mais difícil quando este expõe o indivíduo a consequências aversivas imediatas ou quando o reforço é atrasado (Albuquerque \& Paracampo, 2010).

Quanto à melhor adesão ao uso dos medicamentos, tal resultado pode ser explicado pela própria história de exposição das participantes aos efeitos positivos observados com a administração de tais medicamentos. Por sua vez, o seu uso irregular pode estar relacionado ao bom estado clínico apresentado pelas participantes, ou seja, a ausência de sintomas do LESJ (identificado pela nota zero do SLEDAI de ambas) aumentaria a probabilidade de abandono do uso do medicamento, conforme sugere a literatura sobre o uso irregular de medicamentos em condições crônicas (Otsuki, Clerisme-Beaty, Rand \& Riekert, 2009).

Observou-se que, durante a automonitorização, as participantes, independentemente da condição, apresentaram aumento nos IATs ao serem comparados com os valores obtidos em linha de base. Tais resultados corroboram os encontrados nos estudos de Casseb et al. (2008) e Ferreira e Fernandes (2009), nos quais observou-se aumento nos índices de adesão ao tratamento em adultos com diabetes submetidos a treino de automonitorização. O mesmo ocorreu com os estudos de Hidaka (2007) e Martins et al. (2015), os quais utilizaram crianças com excesso de peso como participantes.

Os resultados obtidos apontaram para uma melhor eficácia quanto à ordem de apresentação dos instrumentos na Condição 2 (RA-R24h), uma vez que os IATs de P2 foram mais elevados do que os de P1. Entretanto, não é possível confirmar tal assertiva em virtude do número reduzido de participantes deste estudo, apontando-se, portanto, a importância da replicação do procedimento em amostras maiores.
Os dados obtidos por meio dos Formulários de RA, combinados com o R24h, demonstraram que, à medida que as participantes foram expostas à análise das condições de controle diário do seu tratamento, em cada turno, os relatos dos comportamentos de adesão aumentaram de frequência. Tais efeitos eram esperados conforme destacado por Barton et al. (1999).

De acordo com a literatura, quando as consequências são remotas, a regra pode não exercer controle efetivo sobre o comportamento do ouvinte. Nesses casos, o controle pode ser mais efetivo se a regra for apresentada por uma autoridade, como o médico reumatologista no tratamento do LESJ, ou quando ocorrem consequências sociais imediatas para o comportamento de seguir a regra, como elogios dispostos pelo profissional contingentes ao relato de seguimento de regras (Albuquerque \& Paracampo, 2010; Ferreira \& Fernandes, 2009; Skinner, 1969). No presente estudo, a terapeuta-pesquisadora dispôs no ambiente reforços sociais na forma de elogios e comentários positivos contingentes aos relatos de cada participante acerca de sua adesão às instruções do tratamento do LESJ.

Apesar de não terem sido realizadas sessões de observação direta dos comportamentos das participantes em contextos de vida diária, ocorreram relatos tanto de adesão quanto de não adesão às instruções do tratamento. Tais resultados sugerem a possibilidade de fidedignidade dos relatos obtidos durante a pesquisa, conforme análise feita por Dunbar-Jacob, Houze, Kramer, Luvster e McCall (2010).

Quanto aos baixos valores dos SLEDAIs e aos bons resultados dos indicadores clínicos obtidos pelas participantes ao início do estudo, observou-se a importância de se utilizarem múltiplas medidas em estudos sobre adesão ao tratamento. Neste caso, não houve correspondência entre as medidas bioquímicas e os relatos de adesão ao tratamento pelas participantes. Ambas estavam com o LESJ controlado, mas, apesar disso, ou mesmo em função disso, apresentaram relatos de não adesão ou de adesão irregular às instruções do tratamento. Tais achados destacam que o uso exclusivo de indicadores bioquímicos pode medir de forma equivocada a adesão ao tratamento, visto que esta pode ser afetada por outros fatores, como características da doença, como parece ser o caso do LESJ - condição crônica, que, mesmo quando inativa, demanda o uso de medicamentos pelo paciente (Dunbar-Jacob et al., 2010).

Desse modo, os resultados deste estudo apontam a necessidade de instalar repertórios de relatos fidedignos em relação aos comportamentos de adesão às instruções prescritas pelo profissional de saúde e emitidos no intervalo entre as consultas. Provavelmente, instrumentos como os utilizados neste estudo auxiliem nesse sentido, o que poderia ser comprovado em pesquisas que repliquem o procedimento utilizado. 


\section{Referências}

Albuquerque, L. C. (2001). Definições de regras. Em H. J. Guilhardi, M. B. B. P. Madi, P. P. Queiroz, \& M. C. Scoz (Orgs.), Sobre comportamento e cognição: Expondo a variabilidade (Vol. 7, pp.132-140). Santo André: ESETec Editores Associados.

Albuquerque, L. C., \& Paracampo, C. C. P. (2010). Análise do controle por regras. Psicologia. USP, 21(2), 253-273. doi: 10.1590/S0103-65642010000200004

American College of Rheumatology (2008). Systemic Lupus Erythematosus (Lupus). Atlanta: American College of Rheumatology.

Barret, D. H., Deitz, S. M., Gaydos, G. R., \& Quinn, P. C. (1987). The effects of programmed contingencies and social conditions on responses stereotypy with human subjects. The Psychological Record, 34, 489-505.

Barton, K. A., Blanchard, E. B., \& Veazey, C. (1999). Selfmonitoring as an assessment strategy in behavioral medicine. Psychological Assessment, 11(4), 490-497. doi : 10.1037/10403590.11.4.490

Bohm, C. H., \& Gimenes, L. S. (2008). Automonitoramento como técnica terapêutica e de avaliação comportamental. Revista Psicolog, 1, 89-101.

Bueno, A. L., \& Czepielewski, M. A. (2010). O recordatório de $24 \mathrm{~h}$ como instrumento na avaliação do consumo alimentar de cálcio, fósforo e vitamina $\mathrm{D}$ em crianças e adolescentes de baixa estatura. Revista de Nutrição, 23(1), 65-73. doi: 10.1590/ S1415-52732010000100008

Casseb, M. S., \& Ferreira, E. A. P. (2012). Treino em automonitoração e comportamentos de prevenção em diabetes Tipo 2. Estudos de Psicologia, 29(1), 135-142. doi: 10.1590/S0103166X2012000100015

Casseb, M. S., Bispo, M. S. M., \& Ferreira, E. A. P. (2008). Automonitoração e seguimento de regras nutricionais em diabetes: dois estudos de caso. Interação em Psicologia, 12(2), 223-233.

Cerutti, D. T. (1994). Compliance with instructions: Effects of randomness in scheduling and monitoring. The Psychological Record, 41, 51-67.

Dunbar-Jacob, J., Houze, M.P., Kramer, C., Luyster, F., \& McCall, M. (2010). Adherence to medical advice: Processes and measurement. In A. Steptoe (Org.), Handbook of Behavioral Medicine: Methods and applications. New York: Springer.

Duvdevany, I., Cohen, M., Minsker-Valtzer, A., \& Lorber, M. (2011). Psychological correlates of adherence to selfcare, disease activity and functioning in persons with systemic lupus erythematosus. Lupus, 20(1), 14-22. doi: 10.1177/0961203310378667

Ferreira, E. A. P., \& Fernandes, A. L. (2009). Treino em autoobservação e adesão à dieta em adulto com diabetes tipo 2 . Psicologia: Teoria e Pesquisa, 25(4), 629-636. doi: 10.1590/ S0102-37722009000400019

Hayes, S. C., Rosenfarb, I., Wulfert, E., Munt, E., Korn, Z., \& Zettle, R. D. (1985). Self- reinforcement effects: An artifact of social standard setting. Journal of Applied Behavior Analysis, 18, 201-214. doi: 10.1901/jaba.1985.18-201
Hidaka, A. H. V. (2007). Tratamento comportamental de crianças com obesidade ou sobrepeso: Estudo exploratório baseado no modelo construcional de I. Goldiamond. (Dissertação de mestrado não publicada). Universidade de Brasília, Brasília, Brasil.

Katz, R. S. (2011). What are the SLEDAI and BILAG evaluations? Recuperado de http://www.lupusil.org/what-are-the-sledaiand-bilag-evaluations.html

Malerbi, F. E. K. (2000). Adesão ao tratamento. In R. Kerbauy, (Org.), Sobre comportamento e cognição. Psicologia comportamental e cognitiva: Conceitos, pesquisa e aplicação, a ênfase no ensinar, na emoção e no questionamento clínico (Vol. 5, pp. 148-155). Santo André, SP: ARBytes Editora.

Malerbi, F. E. K. (2011). Adesão ao tratamento, importância da família e intervenções comportamentais em diabetes. In Sociedade Brasileira de Diabetes (Org.), Diabetes na prática clínica. (módulo 3). Recuperado de http://www.diabetes. org.br/ebook/component/k2/item/53-adesao-ao-tratamentoimportancia-da-familia-e-intervencoes-comportamentaisem-diabetes

Martins, L. C. C. de O., Ferreira, E. A. P., Cavalcante, L. C., \& Almeida, F. P. (2015). Seguimento de regras nutricionais em crianças com excesso de peso. Psicologia: Teoria e Pesquisa, 31(1), 33-41. doi: 10.1590/0102-37722015011465033041

Matos, M. A. (2001). Comportamento governado por regras. Revista Brasileira de Terapia Comportamental e Cognitiva, 3(2), 51-66.

Mulvaney, S. A., Rothman, R. L., Osborn, C. Y., Lybarger, C., Dietrich, M. S., \& Wallston, K. A. (2010). Self-management problem solving for adolescents with type 1 diabetes: Intervention processes associated with an internet program. Patient Education and Counseling, 85, 140-142. doi: 10.1016/j.pec.2010.09.018

Otsuki, M., Clerisme-Beaty, E., Rand, C.S., \& Riekert, K.A. (2009). Measuring adherence to medication regimens in clinical care and research. In S. A. Shumaker, J. K. Ockene, \& K. A. Riekert (Orgs.), The Handbook of health behavior change ( $3^{\mathrm{a}}$ ed., pp. 309-326). New York: Springer Publishing Company.

Palmer, D. L., Osborn, P., King, P. S., Berg, C. A., Butler, J., Butner, J., ... Wiebe, D. J. (2010). The structure of parental involvement and relations to disease management for youth with type 1 diabetes. Journal of Pediatric Psychology, 36(5), 596-605. doi: 10.1093/jpepsy/jsq019

Pongmarutani, T., Alpert, P. T., \& Miller, S. K. (2006). Pediatric systemic lupus erythematosus: Management issues in primary practice. Journal of American Academy of Nurse Practitioner, 18(6), 258-267. doi: 10.1111/j.1745-7599.2006.00128.x

Ribeiro, E. H., Costa, E. F., Sobral, G. M., \& Florindo, A. A. (2011). Desenvolvimento e validação de um recordatório de 24 horas de avaliação da atividade física. Revista Brasileira de Atividade Física e Saúde, 16(2), 132-137.

Sousa, L. M., Paracampo, C. C. P., \& Albuquerque, L. C. (2015). Efeitos de histórias experimentais e de justificativas sociais sobre o comportamento de seguir regras. Psicologia: Reflexão e Crítica, 28(3), 583-592. doi: 10.1590/1678-7153.201528317

Skinner, B. F. (1969). Contingencies of reinforcement: A theoretical analysis. New York: Appleton-Century-Crofts. 
Skinner, B. F. (1974). About behaviorism. New York: Alfred A. Knopf.

Sociedade Brasileira de Reumatologia (2011). Lúpus Eritematoso Sistêmico Juvenil. São Paulo: Sociedade Brasileira de Reumatologia.
World Health Organization (2003). Adherence to long-term therapies: Evidence for action. Genebra: World Health Organization.

Recebido em 28.07.2014

Primeira decisão editorial em26.06.2016

Versão final em 18.08.2016

Aceito em 12.08.2016 